\title{
Research on The Key Technology of Embedded Linux Logistics System
}

\author{
Jianli Chu ${ }^{\mathrm{a}}$, Xia Liub, Jun $\mathrm{Li}^{\mathrm{c}}$, and Xiaoji Chen ${ }^{\mathrm{d}}$ \\ Department of Information Engineering, Xingtai Polytechnic College, Xingtai, P.R. China \\ a397310619@qq.com, b283281266@qq.com, c9693859@qq.com, d1213552916@qq.com
}

Keywords: embedded; Linux; logistics system; key technology; system construction; GSM; RFID

\begin{abstract}
Embedded Linux has a very wide range of applications, but also for the field of logistics information provides a powerful and flexible solution, this paper makes research on the key technology. First of all, research embedded Linux systems build, including the system components, the typical hardware platforms, the kernel cutting, the root file system production, etc; Secondly, research of mobile communication technology, including GSM system composition and GSM in the main application field of logistics information; Finally, research radio frequency identification technologies, including RFID system components and the main technical. With the development of embedded technology for logistics information field gradually mature, The research content of this article will be bound to get a wide range of applications, to promote the development of logistics industry, to improve the level of logistics industry and other aspects of information technology has great significance.
\end{abstract}

\section{Introduction}

Logistics is to point to meet the needs of customers, at the lowest cost, through transport, storage, distribution and so on, to achieve raw materials, semi-finished products, finished products or related information from origin of goods to the commodity consumption in the whole process of planning, implementation and management. The rapid development of the logistics industry, On the one hand, in order to ensure the rapid development of the national economy coordination and stable, played a fundamental role in safeguarding and supporting. On the other hand, it also became important means in the adjustment of industrial structure, changing the mode of economic development and opening up new economic growth point. Logistics industry, the overall level of information technology is relatively backward, unable to adapt to the needs of the rapid expansion of the scale of the logistics industry, embedded technology is the main technical to improve the level of information logistics industry.

Embedded systems is the "control, monitor or assistive devices, machines, and equipment operation device", is centered on application, based on computer technology, software and hardware clipping, suitable for application system for function, reliability, cost, volume, power consumption, etc. Strict requirements, dedicated computer system. Embedded Linux is a Linux operating system that by cutting the increasingly popular Linux operating system and making it in embedded computer systems running on. Embedded Linux inherits the Internet unlimited open source resources, but also has the characteristics of embedded operating system [1]. Embedded Linux has a very wide range of applications, but also provides a powerful flexible solution in logistics information field, in order to achieve effective control and accelerate logistics operation. Embedded Linux technology involved a lot, in this study only four key technologies.

\section{Embedded Linux Systems Build}

\subsection{Embedded System Components}

Embedded system is generally consists of two parts, hardware platform and software platform, shown in Fig 1.

Hardware platform is composed of embedded microcontroller processor and peripheral hardware devices, with the continuous development of chip technology, the embedded processor speeds are 
also increasing, multiprocessor and multi-core processor platform has also been gradually applied in the embedded field, the typical hardware platform shown in Fig 2.

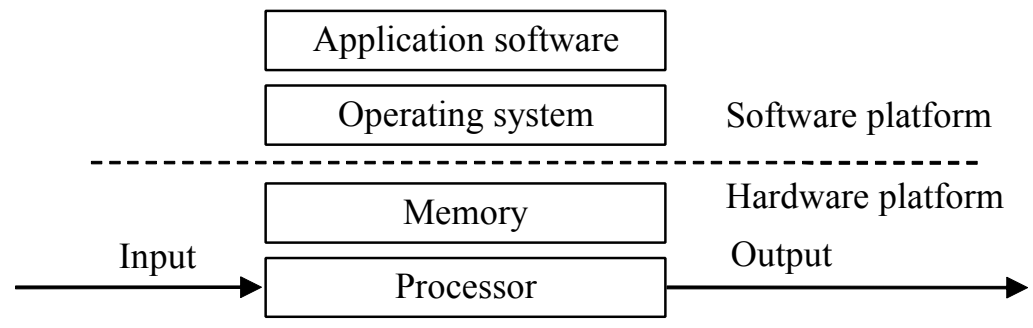

Fig 1. Embedded system components

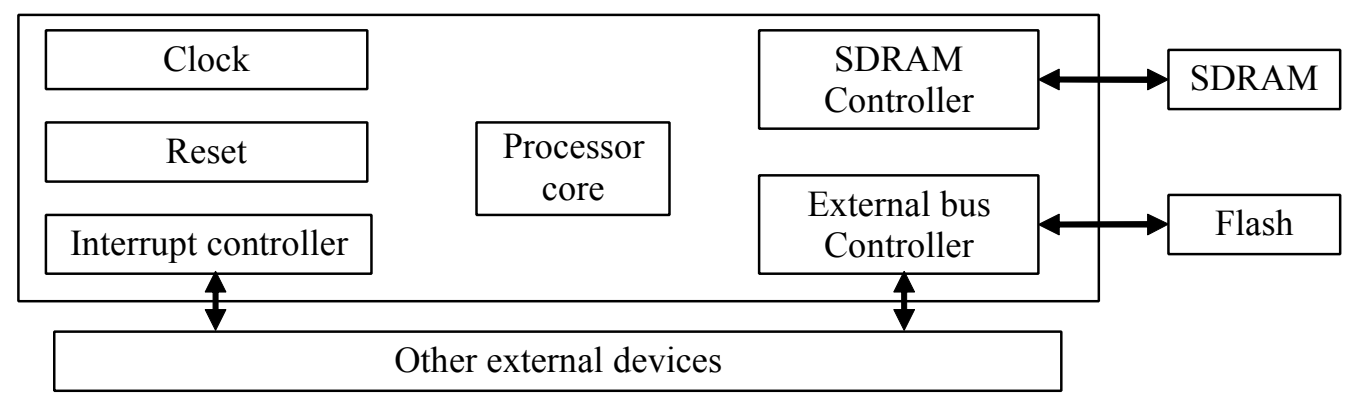

Fig 2. Typical embedded hardware platform

The software platform is composed of embedded operating system and application software. Application software running on embedded operating system, when the processor with the MMU (Memory Management Unit), can be separated from application software and operating system from hardware, compiling and Management, Linux using the separation mechanism, the advantages of high system security, the maintainability is strong, the division of each function module

\subsection{Embedded Linux Kernel Cutting}

First, the kernel configuration. Because there are many versions of the kernel, should according to the specific processor architecture to choose the most suitable kernel version. Once found the appropriate kernel version, unzip it to "\$(PRJROOT $\} /$ kernel" directory. There are four main methods for the configuration of the kernel [4]:

(1) make config: Through the command interface, set each option in turn.

(2) make oldconfig: The configuration based on the original ".config" configuration file, only with option is not set previously encountered that require manual setting.

(3) make menuconfig: Display to "curse"-based, terminal-style configuration menu.

(4) make xconfig: Display based on Tk "X Windows" menu.

Then, compile the kernel. Includes the following three steps:

(1) To establish the dependence of the kernel source code. From the kernel source tree root directory, use "make" command to establish the dependence of the kernel source code.

(2) Set up the kernel image. Can indicate "Makefile" set up "gzip compressed kernel image, also can direct" Makefile" to establish an uncompressed kernel image.

(3) Compile the kernel modules. Run "make" command to compile, the generated image files stored in the "/ arch / arm / boot /" directory, named "zImage".

\section{Mobile Communication Technology}

GSM (Global System for Mobile Communications) is a Global standard for Mobile Communications, GSM terminal within the System through the Service Center can with any GSM terminal or PSTN network terminal for data communication, mainly voice, can provide images and data business [5]. Embedded wireless communication technology can use GSM technology or its improvement. 


\subsection{GSM System Components}

GSM system is mainly composed of mobile station (MS), base station subsystem (BSS) and network subsystem (NSS), as shown in Fig 3 [6]. Base station subsystem (BSS) by base transceiver station (BTS) and the base station controller (BSC); Network subsystem by the mobile switching center (MSC), operation and maintenance center (OMC) and home location register (HLR), visit location register (VLR), authentication center (AUC) and equipment identity register (EIR), etc.

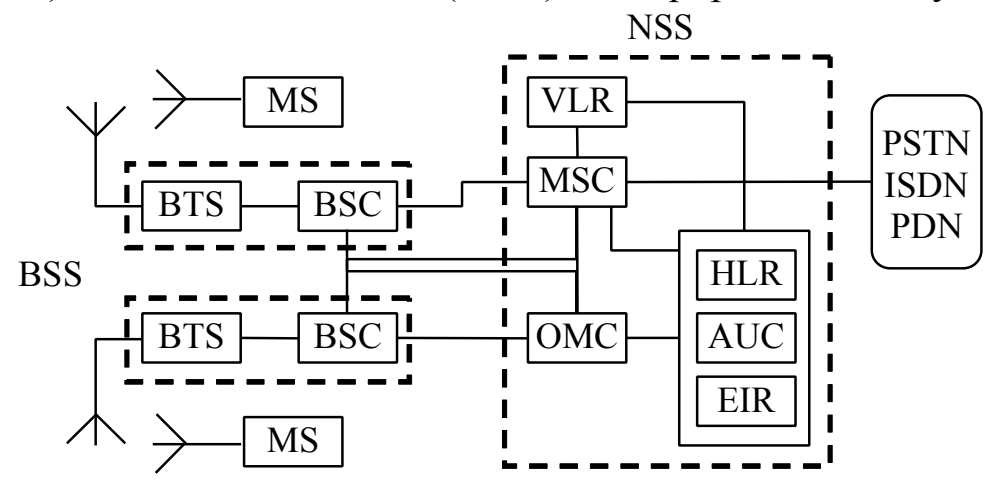

Fig 3. GSM system composition

Each component are described below [7]: MS is a physical device, by subscriber identity module (SIM) and the hardware equipment; BTS includes wireless transmission needs a variety of hardware and software, connection the interface circuit of BSC and BTS itself required for testing and control equipment, etc.; BSC is the BTS and MSC join points, the main function is carries on the wireless channel management, the implementation calls, the communications link established and removed, and control the control of the mobile station within the inter-district switch; MSC is the core of the cellular network, the main function is to control in the MSC region of the user communication control and management; HLR is a database storage local user's location information; VLR is a database storage visiting users location information; AUC identification of user identity, only allow you have the right to access network and access services; OMC is the control and operation of the whole network.

\subsection{GSM Main Application}

The embedded technology has two main applications in the field of logistics information.

(1) Vehicle system. In order to solve the on-board system and ground equipment wireless communication problems, such as large amount of data, slow speed, easy to lost, can use the embedded wireless data communication system [8]. Selects the ARM7 chip, the data deposited in the FLASH memory chip, then export to a serial port by FLASH chips, use the existing network communication. Not only retain the most commonly used serial port, and realize the stable data communication.

(2) Monitoring system. Traditional monitoring systems monitor and control centers via Internet, the public telephone network, or LAN, etc. wired data transmission, constructing the network cost is high, and the monitor position is restricted by the network interface, movement is not flexible. With the improvement of the GSM common network, monitoring system uses the existing wireless mobile network for data transmission, low construction cost, convenient monitor mobile, can achieve arbitrary location monitoring multiple warehouse and carrier.

\section{Radio Frequency Identification Technologies}

Radio Frequency Identification technology (RFID), is a non-contact automatic Identification technology, through Radio Frequency signal automatically identify target and get the relevant data $[9,10]$. Has been widely used in industrial automation, logistics and transportation management, and other fields, become one of hot topics in the study of the current IT industry technology.

\subsection{RFID System Composition}


The most basic RFID consists of three parts, tag, reader and antenna. But in order to make the data transmission, storage and handling of the read, usually RFID also includes two auxiliary parts, middleware and computer system [11]. The system composition structure shown in Fig 4.

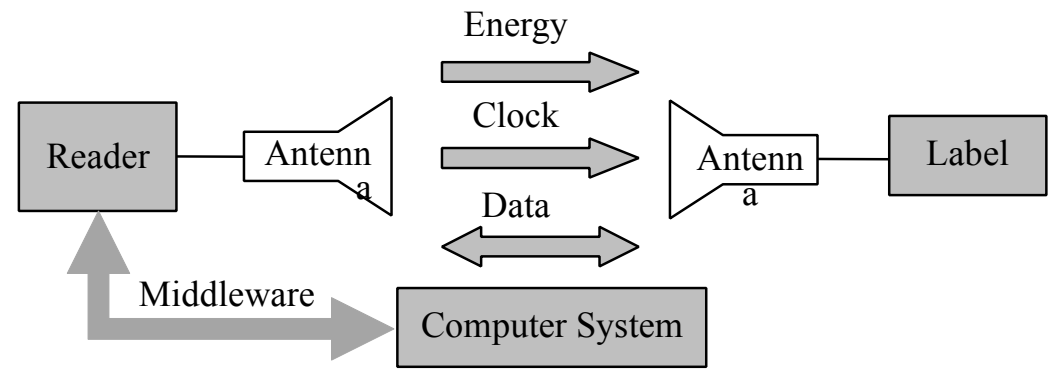

Fig 4. RFID system components

(1) Label. Commonly known as an electronic tag, also calls the transponder, composed of tag chip and the coil, the use of inductive coupling or electromagnetic backscattering coupling principle to realize communication with reader.

(2) Reader. The read / write operation of equipment for RFID tags, including the RF module and digital signal processing unit of the two part, is the most important infrastructure in RFID system.

(3) Antenna. It is an equipment that implementation space RF signal transmission and establish a wireless connection between the RFID tag and reader.

(4) Middleware. It is a software that accept requests from application software, to specify one or more of the reader after launching an operation, receiving, processing to application returns the result data.

(5) Computer system. Is the information management center of the RFID system, by hardware equipment, network equipment, database management systems, software systems and other components.

\subsection{The Main Technology Of RFID}

The RFID application premise, is the key to solve the RFID technology. RFID key technology mainly in the following respects [12]:

(1) RFID systems integration and data management. RFID and wireless communications, sensor networks, information security, industrial control and so on the integration of technology, RFID uses a system of middleware technology, a mass of RFID information resources organization, storage, management, exchange, distribution, data processing and cross-platform computing technology

(2) RFID detection technology and specifications. for different industry applications of RFID tags and related physical features and performance consistency detection technology and the specification, the air interface between tag and reader conformance testing technology and specification, as well as system solutions and comprehensive detection technology and specification.

(3) Multi-object recognition technology. When there are multiple tags within reader signal range, the same time, there are two or more than two tags while return information to the reader, will lead to conflict. Traditional radio technology for air separation multiplex, frequency division multiplexing, time division multiplexing method and code multiplexing method and other methods to solve the similar problems.

(4) Label of energy supply. Active tag comes with batteries and used to power a data carrier; Passive tags required energy obtained from the radio frequency electromagnetic beam. Compared with active radio frequency identification system, passive system requires large transmission power, radio frequency electromagnetic wave on the label via radio frequency (RF) detection, voltage regulator, storage circuit processing, into the tag working voltage needed for the job.

(5) The security and integrity of data transmission. Due to the digital signal will be disturbed in the process of transmission, transmitted to the receiving end may occur a wrong judgment, in order to ensure the integrity of the data, can be used to identify the transmission errors using calibration method, and correct operation. The most commonly used method is parity and redundancy check. 


\section{Conclusion}

Embedded Linux is based on Linux embedded operating system, is widely used in mobile phones, personal digital assistant (PDA), media player and many consumer electronic devices. Embedded Linux has practical significance to the field of logistics information, but has not been widely applied. With the continuous development of logistics enterprises and the embedded technology, embedded technology for logistics information sector will gradually become mature. Embedded Linux has practical significance to the field of logistics information, but has not been widely applied. With the continuous development of logistics enterprises and the embedded technology, embedded technology for logistics information sector will gradually become mature. Through the automatic billing, real-time monitoring, provide a variety of statistical information, reducing staff, inventory management, improve service quality and other means, to optimize business processes, improve logistics efficiency, bring brand-new management idea and service methods for the logistics industry, realize the maximization of enterprise value. Especially logistics digital anti-counterfeiting system, portable storage inventory machine hardware and system software, logistics automation wireless storage information system, embedded experimental logistics system is highly application value and social value, is an important research direction in the future for a period of time.

\section{References}

[1] Baidu Encyclopedia, "Embedded Linux," http://baike.baidu.com/view/1195294.htm , 2013-6-9.

[2] C. Li, J. Xiao, "Application and technology of embedded Linux development," Electronic Industry Press, 2008.

[3] Y. F. Li, W. S. Ou, "ARM Linux embedded system development from entry to the master," Tsinghua University press, 2007.

[4] X. Y. Chen, "Constructing Embedded Systems Based On Linux," Journal of Longyan University, vol. 25, no. 3, pp. 17-21, 2007.

[5] G. Q. Zhao, T. Xing, Y. C. Ren, "Research on Embedded Extend Key Technology in Logistics Control Field," Computer Knowledge and Technology, vol. 6, no. 20, pp. 5615-5617, 2010.

[6] Y. Zhang, "Design and Implementation of the Embedded Logistics Monitor System Based on GSM," Master's Degree of Jiangnan University, 2008.

[7] Vecuronium electronic market, "The main structure of GSM system," www.dzsc.com , 2010-6-7.

[8] Y. Z. Guo, X. Xu, "A design of embedded wireless communication system and its realization," Enterprise technology development, vol. 28, no. 11, pp. 26, 2009.

[9] G. Q. Zhao, T. Xing, Y. C. Ren, "Application of Radio Frequency Identification in Embedded Warehouse Logistics Management," Computer Knowledge and Technology, vol. 6, no. 19, pp. 5339-5341, 2010.

[10] Baidu Encyclopedia, "RFID," http://baike.baidu.com/view/26303.htm?fr=ala0_1, 2013-5-7.

[11]H. Q. Luo, X. W. Xie, "The analysis of the key technology of RFID network," http://www.c114.net, 2013-5-7.

[12] L. Y. Hao, J. P. Zhao, Q. X. Liu, "The Key Technology of RFID and Its Use in the Manage of the Students," Modern Education Technology, vol. 17, no. 1, pp. 80-82, 2007. 